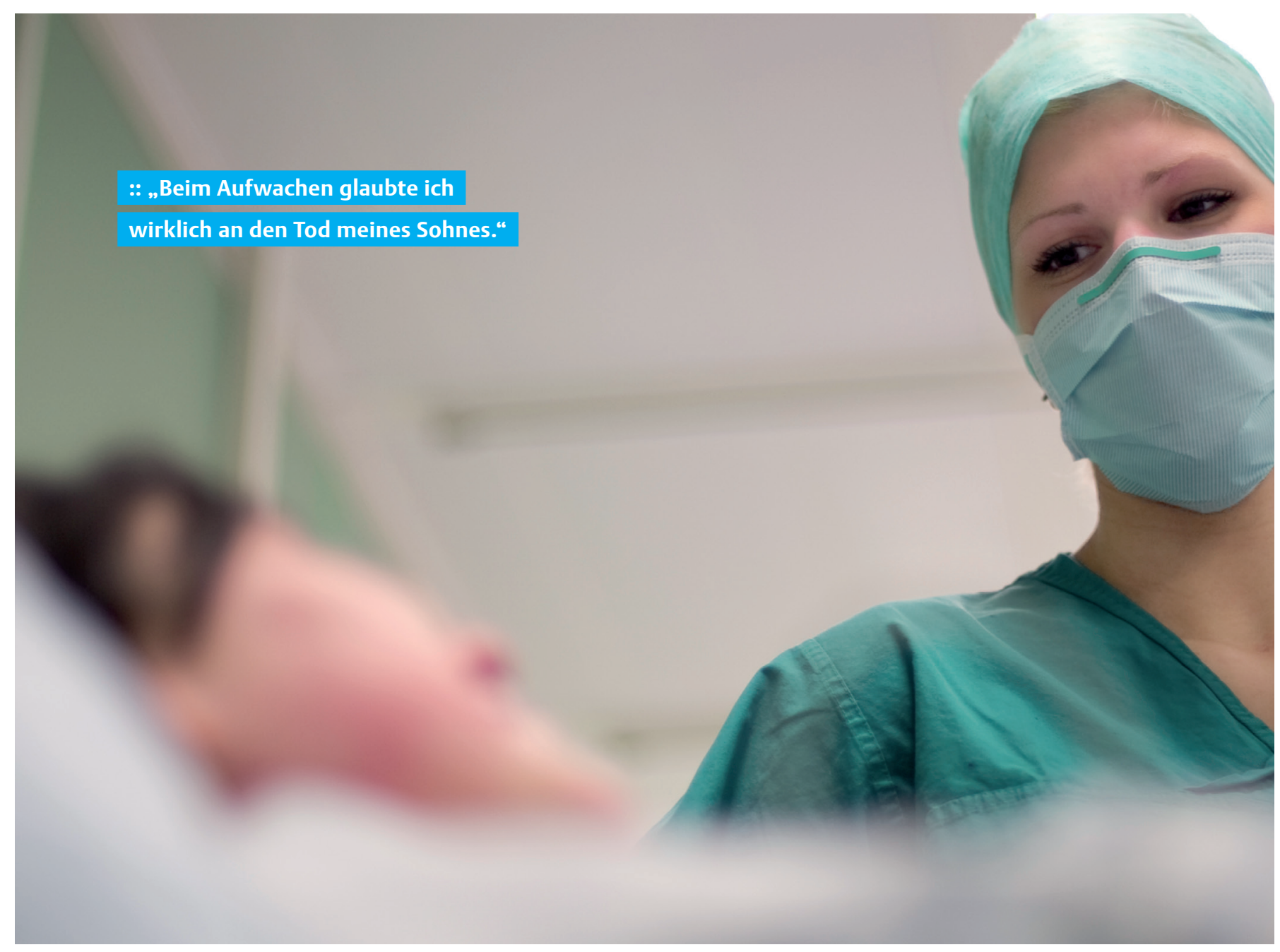

\title{
Eine Intensivpatientin erzählt
}

ERFAHRUNGSBERICHT Wie geht es einem Menschen, der eine lange Zeit auf der Intensivstation verbringen muss? Was erlebt und empfindet er? Im folgenden Interview berichtet eine ehemalige Patientin über ihre Erfahrungen während ihres mehr als zweimonatigen Aufenthalts auf einer Intensivbettenstation. Anlass für das Interview war eine krankenhausinterne Fortbildungsveranstaltung.

Die Patientin (im Folgenden Frau Gruber genannt) war zum Zeitpunkt des Auftretens der Erkrankung 31 Jahre alt, Mutter von zwei Söhnen und arbeitete in einer Bankfiliale in Wien. Die Aufnahmediagnose auf der Intensivstation lautete Guillain-Barré-Syndrom.

Das Interview mit Frau Gruber ist gekennzeichnet durch eine sehr konstruktive und reflektierte Beschreibung des Erleb- ten. Von besonderer Bedeutung ist für Frau Gruber, dass sie sich nicht als belehrender Mensch zeigen möchte und sich darüber im Klaren ist, dass Menschen unterschiedliche Bedürfnisse haben.

- Grüß Gott, Frau Gruber. Zuerst einmal herzlichen Dank für Ihre Bereitschaft, mit mir dieses Interview zu führen und uns an Ihren Erfahrungen und Gefühlen teilhaben zu lassen.
Darf ich Sie bitten, uns Ihren Krankheitsverlauf zu schildern?

Mit 31 Jahren, also im Herbst 2003, be-

kam ich eine Lähmungserscheinung nach einer Virusinfektion. Ich war schon fast zwei Wochen im Krankenstand und hatte unter starken Schmerzen festsitzende Herpesbläschen im Hals und starken Husten. Etwas Nennenswertes werde ich wohl nie vergessen: Ein Schluck Eistee hatte ein ganz seltsames Gefühlserlebnis 
ausgelöst. Ich fror blitzschnell, spürte die Ameisen auf der Schädeldecke kraxeln, runter in den Rücken und zurück rauf spürte ich im Hals, auf der Zunge und in den Kopf hinein ein wanderndes, pelziges, fast schon haariges betäubendes noch nie erlebtes Gefühl, das mich total verwunderte. Beim Abendessen versuchte ich etwas Fleisch zu essen - vergeblich, denn es war mehr ein Würgen. Dann wollte ich ein Glas Wasser trinken - es schoss mir allerdings gleich aus der Nase. Die Lähmung zog sich dann über den Kehlkopf runter. Die Selbstlaute verschwanden beim Sprechen. Meine Kollegin verstand mich am Handy nicht mehr und alarmierte die Rettung.

Ich fühlte mich schon schwach und die Lähmungen waren nun auch in den Fingerspitzen, als ich im Krankenhaus auf der Hautambulanz wegen des brennenden weiß gepunkteten Hautausschlags am Bein landete. Danach kam ich in die HNO-Ambulanz. Man schien ratlos zu sein. Ich war drei Stunden im Krankenhaus unterwegs, danach riss der Film. Teilszenen sind hängen geblieben: Ich war noch fähig, mir den Speichel mittels eines Schlauches abzusaugen. Ich lag im Bett auf der Neurologie und schrieb, was ich sagen wollte, auf einen Zettel nieder. Es war mir unmöglich zu sprechen. Dann fiel mir aber auch bald der Bleistift aus der Hand, ich sah nur noch Doppelbilder, schlief dazwischen immer wieder ein. Permanent wurde ich auf Reflexe kontrolliert, die immer schwächer wurden, und befragt. Eine Ärztin sagte mir, sie hätten einen Verdacht auf Miller-Fisher-Syndrom, das habe auch die Entnahme der Flüssigkeit aus dem Rückenmark ergeben. Ich sei in guten Händen und werde in ein anderes Krankenhaus verlegt, da sei ich sehr gut aufgehoben.

Dort wurde ich direkt auf der Intensivstation aufgenommen. Mir war das allerdings nicht richtig bewusst, die letzten Szenen, die ich mitbekam, waren als man mir die intravenösen Zugänge setzte. Dann wurde ich in den Tiefschlaf versetzt. Das Zwerchfell war gelähmt, die Atmung setzte aus und ich wurde künstlich beatmet. Viele Komplikationen folgten. Zwischen dem Abendessen und dem Einschlafen lagen zwei Tage!
- Sie waren viele Wochen auf der Intensivstation. Einen großen Teil dieser Zeit waren Sie im Tiefschlaf. Wie haben Sie den Aufenthalt auf der Intensivstation und im Besonderen die Aufwachphase erlebt? Meine Erinnerungen bestehen aus dem Teil, den ich in meiner eigenen Welt, also in meinem Kopf, erlebt habe, dem Teil, den ich vermutlich auf der Station durch meine funktionierenden Sinnesorgane wie Fühlen, Hören, Spüren aufgenommen habe und dem Gemischten, das meine Halluzinationen daraus gemacht haben.

Sie waren gefüllt mit Szenen, die mit großer andauernder Angst zu tun hatten, ich wurde verfolgt, man wollte meine Organe, Krankenschwestern unterhielten sich über dubiose Dinge und bedrohten mich, ich hörte Dschungelgeräusche, war immer liegend ans Bett gefesselt.

Mein Sohn starb, weil er in der Welt meiner Phantasie im Auto warten musste, weil gerade keine Besuchszeit war, und er den Motor laufen lassen musste, weil es im Winter ja so kalt war. Er wurde durch die Auspuffgase vergiftet. Zudem konnte dann der rettende Hubschrauber nicht starten, der Defibrillator war defekt.

Ich selbst erstickte qualvoll in einer extrem lang empfundenen Zeitspanne hilflos und starb in einer fremden Gegend unter Krieg, man schlug mir das Telefon aus der Hand, meine letzte Rettung, weil ich ja keine Luft bekam, ich fragte mich ständig: Warum hilft mir denn keiner? Isolation pur ...

Ich erlebte mich an den verschiedensten Orten: im Fischkutter an einem Fluss bei der Oper, mein eigenes Begräbnis, allein liegend am Südbahnhof, eine Wendeltreppe runter holpernd, eine göttliche Stimme, ein helles Licht, dem ich überall hin gefolgt wäre, irre Szenen, die Hollywood-Filmemachern gefallen hätten, die Gefühle jeder Szene wurde x-mal so gewaltig vermittelt wie bei einem normalen Traum, gemischt mit der Umgebung und Menschen aus der Intensivstation, eine „eigene“ Welt, die niemand verstehen kann, der es nicht erlebt hat. Vielleicht mag man es mit den Halluzinationen eines Drogenabhängigen vergleichen.

Zeit gab es nicht. Sie war nicht fassbar. Ich hatte bemerkenswerte Erlebnisse, die so verrückt, aber auch realistisch wa- ren, ich konnte diese nicht hinterfragen, nahm sie so hin und hatte Dinge wie den Hauseinsturz samt meiner Wohnung auch beim Aufwachen in die reale Welt mitgenommen, weil ich nicht differenzieren konnte.

Oft habe ich mir den Fingerkuppelschlauch zum Gerät, das meine Herzfrequenz gemessen hat, runtergearbeitet, um auf mich aufmerksam zu machen, dass ich keine Luft bekam. Manchmal passierte es, dass nicht gleich eine Schwester kam, da war ich oft verzweifelt, der Schleim in meiner Luftröhre war die aller-, allerstärkste Qual für mich, der ich so hilflos ausgeliefert war. Ich dachte oft, ich weiß, dass das letzte Mal, als die Schwester da war, nicht lange her sein musste, aber ich bekam keine Luft! Und das leider häufig. Das Aussaugen des Schleims war zwar unangenehm, aber tat nicht weh und war befreiend.

Beim Aufwachen glaubte ich wirklich an den Tod meines Sohnes und trauerte. Ich musste viel aufarbeiten und beginnen zu differenzieren, was wahr und nicht wahr war. Ich traute der realistischen Welt nicht mehr, fürchtete, dass Augenblicke später wieder alles auf dem Kopf steht. Doch die Bilder fügten sich in der immer wacheren Zeit immer mehr zusammen.

Dann begann die Zeit, in der ich wieder selbst atmete, die Schläuche Stück für Stück aus mir entfernt wurden, ich den ersten Wasserschluck sitzend im Rollstuhl trinken durfte. Jede Bewegung konnte man sich im Kopf vorstellen und kannte man, aber alles war so schwer, wollte

\section{HINTERGRUND}

Im Pschyrembel (257. Auflage) heißt es unter Guillain-Barré-Syndrom: „Meist symmetrisch angeordnete schlaffe Lähmung zunächst der Beine mit Parästhesien, Reflexabschwächung oder Areflexie, eventuell Sensibilitätsstörungen und Muskelatrophie, die (meist innerhalb weniger Tage) bis zur Tetraplegie fortschreiten kann ... bei Mitbeteiligung der Spinalwurzel C4 Gefahr der Atemlähmung, autonome Neuropathie des Herzens mit Rhythmusstörungen, Thrombose in den gelähmten Beinen mit Lungenembolie.“ 
nicht gelingen, ich musste alles lernen. Ganz von vorn. Die Hilfe vom Intensivpflegepersonal empfand ich ganz, ganz positiv. Es war zwar eine Extremerfahrung pur abhängig zu sein, und nicht schön vor allem wenn man mitten im Leben stand! - und sich selbst nackt im Bett liegend zu waschen war eine Stundenaufgabe und sehr unangenehm. Man sprach mich immer bei meinem Nachnamen an, das gab mir das Gefühl, man nimmt mich wahr und wichtig, die Schwestern waren feinfühlig, fragten immer wieder nach meinem Befinden. Kleine Witze bauten mich auf.

Eine der schönsten Erinnerungen ist Heiligabend, der Tag, an dem das Beatmungsrohr aus meinem Hals entfernt wurde und ich das erste Mal wieder sprechen konnte. Die Schwestern und Therapeutinnen halfen zusammen, um mich im Rollstuhl zum Christbaum zu führen, danach machten die Schwestern und ich aus, was ich sagen soll, wenn meine Mutter zur Tür rein kommt. Sie machten diese Weihnachten besonders schön, freuten sich mit mir, das merkte man von Herzen, ich bin so dankbar dafür ...

\section{- Nach den vielen Wochen auf der In- tensivstation mit dem ganzen Auf und $A b$ würde mich nun interessieren: Wie haben Sie den Wechsel von der Intensivstation auf die Bettenstation erlebt?}

Der Wechsel auf die Bettenstation war einerseits befreiend, weil die anderen Intensivpatienten und deren Behandlung mit den Maschinen endlich aus dem Blickwinkel verschwanden, das belastete mich im Wachzustand schon sehr.

Aber der gravierende Unterschied war: Der menschlich einfühlende, fast schon sanfte Umgang der Schwestern fehlte mir. Man fühlt sich auf der Bettenstation plötzlich sich selbst überlassen, verlassen, fast schon hilflos. Diese Zuwendung gibt es nicht auf der Bettenstation. Das war für mich schwer. Hier wäre eine psychologische Unterstützung sehr, sehr wertvoll genau an diesem Punkt.

\section{- Wie ging es nach der Bettenstation weiter?}

Hier kann ich nur noch nach der Entlassung direkt in die Reha einhaken, die
Erfahrung in der Reha war einzigartig und toll, es ging extrem aufwärts.

Der Weg in den Beruf, zurück in die Bankfiliale, war hart, ich hatte kaum Selbstvertrauen, keine Ausdauer, war oft müde, hatte auf Teilzeit gekürzt und von meinem Filialleiter zum Glück jede Unterstützung, doch kaum Verständnis von Kolleginnen und Kollegen, da man mir meine Krankheit nicht primär ansah. Die Kunden fragten oft nach meiner Halsnarbe. Das war auch schwer. Jeder Blick in den Spiegel erinnerte mich an diese Zeit, mit dem Alltagsrhythmus kam die Routine und ich habe heute das Gefühl, ich habe diese Zeit in erster Linie primär einfach verdrängt. Tief runter verdrängt. Bereit zur Verarbeitung meiner Erlebnisse war ich erst vor knapp eineinhalb Jahren, als der Nervenzusammenbruch und das Burnout ausbrachen. Ich lernte in meinen Psychotherapien meine Vergangenheit zu akzeptieren und damit zu leben.

Grundsätzlich werde ich bis heute diese Grundgefühle des Tiefschlafes dieser Angst nicht los, habe oft kein Selbstvertrauen ohne Grund und dieses Urgefühl der Sicherheit verloren. Angst hatte ich davor gehabt, in den ersten acht Monaten eine Art Rückfall zu erleiden, das war für mich lähmend, bei jedem Anzeichen war ich panisch. Heute kann ich viele kleine Besonderheiten in meinem Leben viel mehr schätzen und genießen, ich bin viel ruhiger geworden, brauche auch Ruhe, mag keine Menschenmengen und Lärm seitdem. Komisch war kurz nach dem Spitalsaufenthalt auch das fremde Gefühl, wieder unter Leuten zu sein. Das war eigenartig ...

\section{- Sie sprechen viel über Gefühle: Hat Ihnen das Pflegepersonal bei der Bewälti- gung Ihrer Angst Unterstützung bieten können? Was hat Ihnen geholfen, was hat Ihnen Sicherheit gegeben? \\ Man konnte es vermutlich in meinen Augen erkennen, ich war nachdenklich, traurig, versuchte zu begreifen, zu ver- stehen, was mir die Ärztin zuvor sagte, ich wurde aufgeklärt, dass ich sieben Wochen im Tiefschlaf war. Ich musste weinen. Eine Schwester kam an mein Bett, hielt mir die Hände. Auch wenn sie}

es nicht $100 \%$ ig wissen konnte, sagte sie mir, ich werde alles wieder lernen, alles würde wieder gut, ich solle Geduld haben, und dass ich das erst alles verarbeiten muss - so liebe Worte, sie nahm sich sehr viel Zeit, verschwand nicht gleich wieder hinter einem danebenstehenden PC (die ich übrigens schrecklich finde neben all diesen Geräten und Monitoren, die den ganzen Tag piepsen), sondern sie baute mich mit Worten auf, solange bis ich nicht mehr weinen musste, Zuversicht bekam, dass ich eines Tages doch wieder laufen lernen würde.

Die Worte der Schwester „Ihre beiden Jungs warten doch auch auf Sie zu Hause“ waren da ganz besonders gut, denke ich. Sicherheit hat mir, so komisch es klingen mag, auch die tägliche Beobachtung der Schwestern bei ihrer Arbeit gegeben.

Es war das Besondere, dass man individuell auf mich eingegangen ist und sich auch genau gemerkt hat, was ich erzählt habe oder meine persönlichen Umstände genau kannte, dass ich aus dem Alltag gerissen wurde, man war mit großem Verständnis und Feingefühl für mich da. Auch pflegerisch gesehen. Ich empfand es fast als eine kleine Familie, zu der ich gehörte. Das war das Besondere, würde ich sagen.

Ich muss Ihnen unbedingt dazwischen etwas mitteilen, einfach so, weil mir auch noch so viel im Kopf durchgegangen ist, und vielleicht einiges, was wichtig wäre, ich weiß aber nicht, wo ich das einbauen könnte. Also, zunächst mal möchte ich mich nicht als belehrenden Menschen zeigen. Patienten haben mit Sicherheit verschiedene Erlebnisse und verschiedene Bedürfnisse und ich weiß nicht, ob z. B. ein älterer Mensch überhaupt berührt werden möchte ... Ich mochte es z. B. gern, wenn man bei der Pflege (da war ich schon im „wacheren“ Zustand) bei jedem Schritt, den man tut, auch dazu gesprochen hat, z. B. dass man nicht nur die Hand nimmt und wäscht, sondern mir etwa auch gesagt hat: „So, ich wasch jetzt ihre Hand, dann die Finger, den kleinen bis zum Daumen“, das klingt jetzt irgendwie blöd, aber ich hab mich dabei wohler gefühlt. Ich wusste, was die Schwester jetzt im nächsten Schritt macht, und ich hab zugehört und es hat mich beruhigt. 
- Während der Zeit auf der Intensivstation waren Sie auch lange Zeit beatmet. Wie haben Sie diese Zeit erlebt?

Ich habe zu Beginn gar nicht bemerkt, dass ich bei der Atmung künstlich unterstützt wurde, erst als ich so im Halbwachzustand meine Mama sagen hörte, dass es so sei und ich nach dem Schlauch griff. Ich wunderte mich zu Beginn, dass ich das gar nicht spürte und war voll der Meinung, ich hab doch selbst geatmet ... Da war kein Unterschied zu erkennen. Es war, glaub ich, doch gerade zu Beginn eine Zeit, wo ich nicht verschleimt war, so glaub ich das heute, weil ich auch später eben oft das Problem hatte, besonders vielleicht im wachen Zustand, mit dem Schleim zu atmen, der sich halt mit jedem Atemzug rauf und runter bewegte, mal mehr, mal weniger. Wenn ich Glück hatte gar nicht, das war schön. Dann musste ich mir fast nicht überlegen, ob ich mir diese Fingerspitze vom Zeigefinger zupfte, anders konnte ich nicht um Aussaugen bitten ... Man war so unglaublich abhängig, das war eine große Stresssituation. Es war z.B. oft eine Schwester, die immer wieder kam, und ich war so froh darüber, dass sie Verständnis zeigte - doch einmal ist es mir auch passiert, dass eine Schwester sagte, dass das nicht immer ginge, „nicht schon wieder", ich war dann schon frustriert. Aber das war Gott sei Dank sehr selten. Man hat schon Angst, dass man vielleicht den Eindruck auf das Personal macht, man wolle Aufmerksamkeit oder nervt vielleicht absichtlich, aber das war keineswegs der Fall. Ich war froh, wenn ich Luft bekam und schlafen konnte, ich war so unglaublich erschöpft.

Ich bekam von den Schwestern oft das Feedback, dass ich eine ruhige, brave Patientin, eine sehr umgängliche gewesen sei, ich freute mich natürlich darüber, aber ich möchte auch offen und ehrlich sagen: Leise kam in mir auch der Gedanke auf, was ist, wenn man ein anstrengender Patient ist ...

\footnotetext{
- Falls es für Sie möglich ist, wäre es großartig, wenn Sie ein paar wenige ganz konkrete Maßnahmen nennen könnten, von denen Sie glauben, dass es ganz wichtig ist, dass die Pflegenden das in der täglichen Arbeit berücksichtigen.
}

Also, ich glaube, dass es ganz wichtig ist, dass man den Patienten sanft berührt und darauf achtet, was man in seiner Umgebung sagt. Also eventuell keine privaten Gespräche in der unmittelbaren Umgebung oder auch eher positive Wörter, ich bin nach meinem Erlebnis davon überzeugt, dass der Patient - auch wenn er schläft - Berührungen, Worte und kurze Bilder durch Augenöffnen genau spürt und in seine Welt aufnimmt.

Ich glaube, es wäre wichtig, auf die Reaktionen des Patienten genau zu achten, ich soll im Schlaf geweint haben oder mit den Armen um mich geschlagen haben, ich denke, dass ich da etwas Heftiges oder Schlimmes geträumt habe und glaube, dass es in dieser Situation wichtig wäre, nicht nur festzuhalten, sondern auch beruhigende Worte auszusprechen, sanfte Worte, streichelnde Berührungen.

Ich glaube, eine reine ausführende pflegende Maßnahme spürt man als Patient schon. Irgendwie scheint es eine Antenne zu geben, auch wenn man schläft.

In der Aufwachphase strengten mich auch kurze Besuche Angehöriger sehr an und die aus dem Radio schallende Musik, jeder Ton schmerzte in meinem Kopf, ich konnte nichts tun. Ich glaube, dass Musik überbewertet wird, sie tut vielleicht nicht immer gut, das erkennt man am besten am Patienten.

In der Zeit des Aufwachens war ich wie geschockt über das, was passiert war, verwundert ... Unsicherheit, wie weit die Rückbildung der Lähmung geht, Angst, ohne Geräte einfach zu sterben, keine Orientierung, keine Kontrolle über meine Bewegungen, die ich neu erlernte - diese Angst wurde durch Zuspruch von Schwestern und Ärzten und auch Nähe beendet. Besonders wichtig war für mich auch Einfühlungsvermögen, weil man sensibler ist. Vielleicht ist es auch irgendwie wichtig, dass nach dem Aufwachen ein Angehöriger auch erzählt, was sich zu Hause tut.

Als mir meine Mama von den Jungs erzählt hat, dass sie auf mich warten und es ihnen gut geht, habe ich immer wieder unter Tränen versucht ihr mitzuteilen, dass sie mich anlügt. Dass sie mich nicht belügen soll, weil sie mich schonen möchte. Ich konnte nicht zur wachen Welt unterscheiden. Sie agierte und holte die Jungs zum Besuch. Erst dann wurde mir klar, ach Gott, was läuft da ... Ich dachte über die Situation nach, und der Genesungsprozess hing sehr viel davon ab, glaube ich! Erst als ich wusste, dass es meinen Kindern gut geht, konnte ich mich wieder auf mich konzentrieren, darauf, wieder nach Hause zu kommen, und der Wille war einfach auch wieder da. Vielleicht ist das vielen Angehörigen nicht so klar. Man sollte dem Patienten gut zuhören, ob er irgendwelche Dinge von sich gibt, die ihn aufregen, und man sollte dann abklären, ob das auch der Realität entspricht.

Wichtig ist auch zu berücksichtigen, dass es sehr schwer ist, plötzlich so hilflos und abhängig zu sein. Ich glaube, großes Verständnis und eine gewisse Stärke aufseiten der Pfleger sind Voraussetzung für diesen sicher sehr anstrengenden Beruf.

Aufgrund des Datenschutzes und um die Anonymität dieser Frau zu wahren, wurden der Name der Patientin und Textpassagen so verändert, dass ein Wiedererkennen der Person nicht möglich ist. Das Interview fand aufgrund von Praktikabilität per Mailverkehr statt. Der hier abgedruckte Teil ist ein Auszug aus dem gesamten Interview, das im Mai 2011 von Martin Fangmeyer durchgeführt wurde.

\section{AUTOR \\ Martin Fangmeyer} ist diplomierter Gesundheitsund Krankenpfleger mit Sonderausbildung Intensivpflege und hat auf verschiedenen Intensivstationen in Wien gearbeitet. Seit 2006 ist er Pflegeberater mit Schwerpunkt neurologische Intensivpflege, Onkologie, Strahlentherapie und Palliative Care.

E-Mail: Martin.Fangmeyer@wienkav.at

\section{BIBLIOGRAFIE}

DOI 10.1055/s-0032-1304778

Intensiv 2012; 20 (2): 69-72

(c) Georg Thieme Verlag KG

Stuttgart · New York · ISSN 0942-6035 